\title{
ESTILOS DE VIDA Y SU RELACIÓN CON LAS CARACTERÍSTICAS SOCIO DEMOGRÁFICAS EN ESTUDIANTES DE LA FACULTAD DE ENFERMERIA DE LA UNIVERSIDAD NACIONAL SAN LUIS GONZAGA DE ICA, 2015.
}

Lifestyles and their relation with the socio demographic characteristics in students of the Faculty of Nursing of the National University San Luis Gonzaga of Ica, 2015

\section{Angélica Villarroel Huamaní $i^{1, a, b, c}$, Amparo Saravia Cabezudo ${ }^{2, a, c, d}$, Rosa Castillo Paredes ${ }^{3, a, e}$}

1. Facultad de Enfermería - Universidad Nacional San Luis Gonzaga de Ica, Perú.

2. Dirección General de Salud, Ica, Perú.

3. Hospital Regional Ica, Perú.

a Licenciada en Enfermería, b Enfermera Especialista en Administración de los Servicios de Salud, c Magister en Educación con mención en la Administración y Planificación de la Educación Superior, d Enfermera Especialista en Salud Publica, e Magister en Salud Publica.

\section{RESUMEN}

El estilo de vida es uno de los mayores condicionantes sobre el estado de salud de la población, la adopción generalizada de un estilo de vida saludable podría reducir la mortalidad global. Objetivo: Establecer la relación entre los estilos de vida y las características socio demográficas de los estudiantes de la Facultad de Enfermería de la Universidad Nacional San Luis Gonzaga de Ica en 2015. Material y métodos: Investigación aplicada, de tipo descriptivo, diseño observacional, de corte transversal, en 200 alumnos seleccionados mediante muestreo no probabilístico por cuotas de $25 \%$ por cada año de estudios, mediante entrevista auto aplicada se empleó un cuestionario estructurado para la investigación, se utilizó estadística descriptiva y como prueba de hipótesis el reactivo $X^{2}$ a un nivel de significancia $p<0,05$. Resultados: la muestra estuvo constituida por estudiantes $>20$ años de edad $(64,0 \%)$, de sexo femenino $(82,0 \%)$; solteros $(96,5 \%)$, sin hijos $(88,5 \%)$; estudios exclusivos $(70,5 \%)$; ingreso familiar mensual >S/. 1000.00 n. s. (57,0\%), el 90,5\% presentaron estilos de vida adecuados caracterizándose principalmente por tener más de 20 años $(62,4 \%)$, pertenecer al sexo femenino $(85,6 \%)$, estado civil soltero $(96,7 \%)$, no tener hijos $(87,8 \%)$, estudiar exclusivamente $(68,0 \%)$; tener un ingreso familiar mensual mayor a S/. 1000.00 n. s. $(54 ; 1 \%)$ y estar cursando el $5^{\circ}$ año de estudios $(22 ; 1 \%)$, la prevalencia de vida de relaciones sexuales fue de $38,5 \%$ y de prácticas sexuales adecuadas de 61,0\%; con similares características al de los estilos de vida globales. Conclusiones: se concluye que existe relación en el tipo de estilos de vida y las características sociodemográficas incluidas en el estudio, con una alta tasa de conductas adecuadas.

Palabras claves: Estilos de vida, características sociodemográficas, estudiantes enfermería.

\section{SUMMARY}

The lifestyle is one of the biggest constraints on the state of health of the population, the widespread adoption of a healthy lifestyle could reduce overall mortality. Objective: To establish the relationship between lifestyles and demographic partner of students of the Faculty of Nursing at the National San Luis Gonzaga Ica University in 2015 features. Methods: Applied research, descriptive, observational design, cross-sectional, 200 students selected by non-probability quota sampling of $25 \%$ for each year of study; job interview self-administered a structured questionnaire research; Descriptive statistics was used as the reagent test hypotheses $X 2$ to a significance level of $p<0,05$. Results: The sample consisted of students> 20 years of age $(64.0 \%)$; female $(82.0 \%)$; single $(96.5 \%)$; without children (88.5\%); exclusive studies (70.5\%); monthly family income > S /. 1000.00 n. s. (57.0\%); $90.5 \%$ had lifestyles that characterized mainly, have more than 20 years $(62.4 \%)$; being female 
(85.6\%); single marital status (96.7\%); not having children (87.8\%); exclusively study (68.0\%); have a higher monthly family income of S $/ .1000 .00 \mathrm{n}$. s. (54.1\%) and to be attending the 5 th year of studies (22.1\%); the lifetime prevalence of sex was $38.5 \%$ and $61.0 \%$ adequate, with similar characteristics to that of global lifestyles sexual practices. Conclusions: We conclude the correlation between the type of lifestyle and sociodemographic characteristics included in the study, with a high rate of appropriate behaviors.

Keywords: Lifestyle, sociodemographic characteristics, nursing students.

\section{INTRODUCCION}

En los últimos años en todo el mundo se están observando cambios negativos en el comportamiento de la población joven, siendo más prevalente las alteraciones en los hábitos alimenticios, la actividad física y la utilización del tiempo libre; estas modificaciones se traducen principalmente en una mala alimentación, sedentarismo y abusos en el consumo de bebidas alcohólicas, tabaco y drogas ilegales, muchos de ellos impuestos por la globalización y los medios de comunicación; todos aquellos comportamientos, han sido demostrados científicamente como determinantes de riesgo para enfermedades transmisibles y no transmisibles asociadas a estilos de vida no saludables (1). Rizo M, Baeza M. Concluyen que a pesar de los niveles de sobrepeso y obesidad entre los universitarios es menor que el reportado en la población general, existen desequilibrios en su alimentación, el consumo de macro y micro nutrientes es deficiente, lo que indica que el tener conocimientos de nutrición, no influye en la toma de decisiones para una alimentación y estilo de vida saludables (2). Irazusta A y colaboradores. Concluyen que los encuestados tienen una gran preocupación por la imagen corporal, pero la forma de "cuidar" su cuerpo es a través de dietas deficitarias y desequilibradas, en lugar de hábitos saludables como una dieta equilibrada y actividad física sistemática, por lo que consideran recomendar, incluir $y / 0$ reforzar en los planes de estudio de enfermería los contenidos sobre hábitos saludables en relación a la alimentación y la actividad física ya que serán los profesionales de enfermería los encargados de trasmitir al resto de la población(3). Laguado $\boldsymbol{J} \boldsymbol{y}$ colaboradores. Concluyen que, en la etapa universitaria diferentes factores interfieren el mantener una vida saludable; en los estudiantes de Enfermería del estudio, los hábitos saludables no son realizados de forma rutinaria. Considerándose estos como no saludable según lo propuesto por Nola Pender (4). Ponte A. (Tesis: Pre Grado) Concluye. Que los estudiantes de Enfermería tienen un estilo de vida saludable, según dimensiones predomina en lo saludable: nutrición saludable, crecimiento espiritual y relaciones interpersonales. $Y$ en lo no saludable: actividad física y salud con responsabilidad en los cinco años de estudio, a diferencia de la dimensión gestión de tensiones donde practican lo no saludable, sólo los estudiantes de segundo, tercero y quinto año, practican técnicas de relajación por 20 minutos (5). Nuestra sociedad es altamente sensible a la adopción de nuevos patrones de consumo, los cuales son puestos en práctica muy rápidamente por nuestra población; la población joven en pleno en su desarrollo personal y educativo accede a instituciones de formación técnica y universitaria, en las cuales en interacción con sus pares suelen adoptar muchos de los estilos de vida negativos para su salud, y por lo tanto luego de algún tiempo desarrollan daños con una alta tasa de cronicidad y deterioro de la calidad de vida.

El personal de enfermería por la propia naturaleza de su trabajo en los servicios de Salud Pública, tiene un enorme potencial para realizar actividades de promoción de los estilos de vida saludables en la población con la que 
interactúa durante las actividades comunitarias; el contacto con los jóvenes y sus familiares le permite tener un tiempo útil $y$ suficiente para poder fomentar la adopción de los mismos, que permitan prevenir enfermedades crónicas, discapacidad y muerte (6). Señalándose como Objetivo del estudio. Establecer la relación entre los estilos de vida y las características socio demográficas de los estudiantes de la Facultad de Enfermería de la Universidad Nacional San Luis Gonzaga de Ica. Año 2015. Con la finalidad de identificar probables factores de riesgo, y líneas de trabajo para futuras intervenciones de promoción de la salud, de prevención y control de los estilos de vida no saludables del personal de enfermería.

\section{MATERIAL Y METODOS}

Investigación aplicada, tipo descriptivo, diseño observacional, de corte transversal. La población estuvo conformada por alumnos de la facultad de Enfermería de la Universidad Nacional San Luis Gonzaga de Ica. El estudio se realizó en la Facultad de Enfermería de la Universidad Nacional San Luis Gonzaga de Ica, ubicada en la periferia del cercado de la ciudad de lca y que en el presente año cuenta con una población total aproximada de 520 alumnos matriculados distribuidos en 5 años académicos y 10 secciones. La muestra estuvo conformada por 200 estudiantes, incluyéndose en el estudio 40 alumnos (25\% del total de la muestra) por cada año de estudios. Se aplicó un muestreo no probabilístico por cuotas, teniendo como unidad de muestreo el aula de clases según años de estudio.

Se incluyeron alumnos de la Facultad de Enfermería de la Universidad Nacional San Luis Gonzaga de Ica matriculados y presentes en la institución educativa al momento del estudio y aceptar a participar voluntariamente en la aplicación del cuestionario. Se excluyeron a los estudiantes que no aceptaron participar en el estudio estando presentes y a los que estuvieron ausentes al momento de la aplicación del cuestionario.

\section{RESULTADOS}

En la presente investigación se logró entrevistar 200 alumnos de la Facultad de Enfermería de la Universidad Nacional San Luis Gonzaga de Ica. La muestra estuvo constituida en su mayoría por estudiantes mayores de 20 años de edad con el 64,0\% (128 estudiantes), de sexo femenino con el $82,0 \%$ (164 estudiantes), solteros con el $96,5 \%$ (193 estudiantes), sin hijos con el 88,5\% (177 estudiantes), que estudian exclusivamente con el $70,5 \%$ (141 estudiantes) y con un ingreso familiar mensual >S/. 1000,00 nuevos soles con el $57,0 \%$ (114 estudiantes), la muestra se distribuyó equitativamente entre los cinco años de estudio con el 25\% (40 estudiantes) cada uno. Tabla No1

Tabla № 01: Distribución porcentual de los encuestados según variables de estudio.

\begin{tabular}{|c|c|c|}
\hline Variables & \multicolumn{2}{|c|}{$N=200$} \\
\hline Grupo etario (años). & $\mathrm{n}$ & $\%$ \\
\hline$<20$ & 72 & 36,0 \\
\hline$>20$ & 128 & 64,0 \\
\hline Sexo. & $\mathrm{n}$ & $\%$ \\
\hline Masculino & 36 & 18,0 \\
\hline Femenino & 164 & 82,0 \\
\hline Estado civil. & $\mathrm{n}$ & $\%$ \\
\hline Soltero & 193 & 96,5 \\
\hline Casado/unión libre & 7 & 3,5 \\
\hline Tiene hijo(s). & $\mathrm{n}$ & $\%$ \\
\hline Sí & 23 & 11,5 \\
\hline No & 177 & 88,5 \\
\hline Trabaja además de estudiar. & $\mathrm{n}$ & $\%$ \\
\hline Sí & 59 & 29,5 \\
\hline No & 141 & 70,5 \\
\hline Ingreso familiar mensual (S/.). & $\mathrm{n}$ & $\%$ \\
\hline$<S / .1000,00$ & 86 & 43,0 \\
\hline$>S / .1000,00$ & 114 & 57,0 \\
\hline Año de estudios & $\mathrm{n}$ & $\%$ \\
\hline Primero & 40 & 20,0 \\
\hline Segundo & 40 & 20,0 \\
\hline Tercero & 40 & 20,0 \\
\hline Cuarto & 40 & 20,0 \\
\hline Quinto & 40 & 20,0 \\
\hline
\end{tabular}

Fuente: Base de datos.

Tabla $\mathbf{N}^{\circ}$ 02. Se pudieron determinar diferencias estadísticas altamente significativas $(p<0,05)$ entre las categorías de la variable. De acuerdo a la clasificación de los estilos de vida de los estudiantes encuestados se pudo determinar que el 90,5\% (181 
estudiantes), presentaban estilos de vida adecuados, mientras que el 9,5\% (19 estudiantes) restantes presentaron estilos de vida inadecuados.

Tabla Nº2: Distribución porcentual de los encuestados según clasificación de estilos de vida.

\begin{tabular}{ccc}
\hline Variables & \multicolumn{2}{c}{$\mathrm{N}=200$} \\
\hline Clasificación & $\mathrm{n}$ & $\%$ \\
\hline Inadecuadas & 19 & 9,5 \\
Adecuadas & 181 & 90,5 \\
\hline Total & 200 & 100,0 \\
\hline Fuente: Base de datos. & $\mathrm{X} 2: 46,69 ; \mathrm{gl}: 1 ; \mathrm{p}<0,05$.
\end{tabular}

Tabla $\mathbf{N}^{\circ}$ 03. En el análisis bifactorial entre los tipos de estilos de vida y las variables de estudio, resultaron estadísticamente significativas las categorías: estilos de vida adecuados y sexo femenino $(p<0,5)$, estilos de vida adecuado y no trabajar $(p<0,05)$, estilos de vida adecuados e ingreso familiar mensual $>S / .1000,00$ nuevos soles $(p<0,05)$ y estilos de vida adecuados y cursar el último año de estudios $(p<0,05)$.

Tabla № 03: Distribución porcentual de los encuestados según clasificación de estilos de vida y variables de estudio.

\begin{tabular}{|c|c|c|c|c|c|}
\hline \multirow{3}{*}{$\begin{array}{l}\text { Variables } \\
\text { Grupo etario } \\
\text { (años). }\end{array}$} & \multicolumn{4}{|c|}{$\mathrm{N}=200$} & \multirow{3}{*}{$\begin{array}{c}\text { Prueba de } \\
\text { hipótesis }\end{array}$} \\
\hline & \multicolumn{2}{|c|}{$\begin{array}{c}\text { Inadecuadas } \\
\mathrm{n}=19\end{array}$} & \multicolumn{2}{|c|}{$\begin{array}{c}\text { Adecuadas } \\
n=181\end{array}$} & \\
\hline & $\mathrm{n}$ & $\%$ & $\mathrm{n}$ & $\%$ & \\
\hline$<20$ & 4 & 21,1 & 68 & 37,6 & 2,04 \\
\hline$>20$ & 15 & 78,9 & 113 & 62,4 & $\mathrm{gl}: 1 ; p>0,05$ \\
\hline Sexo. & $\mathrm{n}$ & $\%$ & $\mathrm{n}$ & $\%$ & $\mathrm{X}^{2}$ \\
\hline Masculino & 10 & 52,6 & 26 & 14,4 & 17,06 \\
\hline Femenino & 9 & 47,4 & 155 & 85,6 & $g l: 1 ; p<0,05$ \\
\hline Estado civil. & $\mathrm{n}$ & $\%$ & $\mathrm{n}$ & $\%$ & $\mathrm{X}^{2}$ \\
\hline Soltero & 18 & 94,7 & 175 & 96,7 & 0,19 \\
\hline $\begin{array}{l}\text { Casado/unión } \\
\text { libre }\end{array}$ & 1 & 5,3 & 6 & 3,3 & $g l: 1 ; p>0,05$ \\
\hline Tiene hijo(s). & $\mathrm{n}$ & $\%$ & $\mathrm{n}$ & $\%$ & $\mathrm{X}^{2}$ \\
\hline Sí & 1 & 5,3 & 22 & 12,2 & 0,80 \\
\hline No & 18 & 94,7 & 159 & 87,8 & $\mathrm{gl}: 1 ; p>0,05$ \\
\hline $\begin{array}{l}\text { Trabaja además } \\
\text { de estudiar. }\end{array}$ & $\mathrm{n}$ & $\%$ & $\mathrm{n}$ & $\%$ & $X^{2}$ \\
\hline Sí & 1 & 5,3 & 58 & 32,0 & 5,93 \\
\hline No & 18 & 94,7 & 123 & 68,0 & $g l: 1 ; p<0,05$ \\
\hline $\begin{array}{l}\text { Ingreso familiar } \\
\text { mensual (S/.). }\end{array}$ & $\mathrm{n}$ & $\%$ & $\mathrm{n}$ & $\%$ & $\mathrm{X}^{2}$ \\
\hline$<S / .1000,00$ & 3 & 15,8 & 83 & 45,9 & 6,34 \\
\hline$>S / .1000,00$ & 16 & 84,2 & 98 & 54,1 & $g l: 1 ; p<0,05$ \\
\hline Año de estudios & $\mathrm{n}$ & $\%$ & $\mathrm{n}$ & $\%$ & $\mathrm{X}^{2}$ \\
\hline Primero & 2 & 10,5 & 38 & 21,0 & 16,52 \\
\hline Segundo & 3 & 15,8 & 37 & 20,4 & gl:5; $p<0,05$ \\
\hline Tercero & 4 & 21,1 & 36 & 19,9 & \\
\hline Cuarto & 10 & 52,6 & 30 & 16,6 & \\
\hline Quinto & 0 & 0,0 & 40 & 22,1 & \\
\hline
\end{tabular}

Dentro de los alumnos que reconocieron presentar estilos de vida adecuados tenemos (181 estudiantes), se observaron que las características más frecuentes fueron: tener más de 20 años 113 estudiantes (62,4\%), pertenecer al sexo femenino 155 estudiantes $(85,6 \%)$, estado civil soltero 175 estudiantes $(96,7 \%)$, no tener hijos 159 estudiantes $(87,8 \%)$, estudiar exclusivamente 123 estudiantes (68\%), tener un ingreso familiar mensual mayor a S/. 1000,00 nuevos soles 98 estudiantes $\left(54,1 \%\right.$ ) y estar cursando el $5^{\circ}$ año de estudios 40 estudiantes (22,1\%).

Tabla $\mathbf{N}^{\circ}$ 04. Se observaron diferencias estadísticas significativas entre las categorías de la variable $(p<0,05)$. El $38,5 \%$ (77 de los alumnos) de la Facultad de Enfermería reconocieron tener o haber tenido relaciones sexuales en su vida, mientras que el $61,5 \%$ (123 estudiantes) refirieron no tener ni haber tenido actividad sexual.

Tabla N 04: Distribución porcentual de los encuestados según actividad sexual.

\begin{tabular}{lll}
\hline Relaciones sexuales. & $\mathrm{n}$ & $\%$ \\
\hline Sí & 77 & 38,5 \\
No & 123 & 61,5 \\
\hline Total & 200 & 100,0 \\
\hline Fuente: Base de datos & & \\
$X^{2}: 10,58 ;$ gl: $1 ; p<0,05$. & &
\end{tabular}

Tabla $\mathbf{N}^{\circ}$ 05. Los alumnos sexualmente activos (77 estudiantes), se caracterizaron principalmente por tener más de 20 años de edad 57 estudiantes $(74,0 \%)$, ser de sexo femenino 54 estudiantes $(70,1 \%)$, de estado civil soltero 70 estudiantes $(90,9 \%)$, no haber tenido hijos 62 estudiantes (80,5\%), estudiar exclusivamente 50 estudiantes $(64,9 \%)$, tener un ingreso familiar mensual mayor a $\mathrm{S} /$. 1000,00 nuevos soles 52 estudiantes $(67,5 \%)$ y estar cursando el $5^{\circ}$ año de estudios 31 estudiantes $(40,3 \%)$. 
Tabla № 05: Distribución porcentual de los encuestados sexualmente activos según variables de estudio.

\begin{tabular}{|c|c|c|}
\hline \multirow{2}{*}{$\begin{array}{l}\text { Variables } \\
\text { Grupo etario (años). }\end{array}$} & \multicolumn{2}{|c|}{$N=77$} \\
\hline & $\mathrm{n}$ & $\%$ \\
\hline$<20$ & 20 & 26,0 \\
\hline$>20$ & 57 & 74,0 \\
\hline Sexo. & $\mathrm{n}$ & $\%$ \\
\hline Masculino & 23 & 29,9 \\
\hline Femenino & 54 & 70,1 \\
\hline Estado civil. & $\mathrm{n}$ & $\%$ \\
\hline Soltero & 70 & 90,9 \\
\hline Casado/unión libre & 7 & 9,1 \\
\hline Tiene hijo(s). & $\mathrm{n}$ & $\%$ \\
\hline Sí & 15 & 19,5 \\
\hline No & 62 & 80,5 \\
\hline Trabaja además de estudiar. & $\mathrm{n}$ & $\%$ \\
\hline Sí & 27 & 35,1 \\
\hline No & 50 & 64,9 \\
\hline Ingreso familiar mensual (S/.). & $\mathrm{n}$ & $\%$ \\
\hline$<S / .1000,00$ & 25 & 32,5 \\
\hline$>S / .1000,00$ & 52 & 67,5 \\
\hline Año de estudios & $\mathrm{n}$ & $\%$ \\
\hline Primero & 6 & 7,8 \\
\hline Segundo & 15 & 19,5 \\
\hline Tercero & 3 & 3,9 \\
\hline Cuarto & 22 & 28,6 \\
\hline Quinto & 31 & 40,3 \\
\hline
\end{tabular}

Fuente: Base de datos.

Tabla $\mathbf{N}^{\circ}$ 06. En el análisis bifactorial entre los tipos de relaciones sexuales y las variables de estudio, resultaron estadísticamente significativas las categorías, conductas sexuales adecuadas y tener hijos $(p<0,05)$ y conductas sexuales adecuadas y cursar el cuarto año de estudios $(p<0,05)$.

Mientras que los alumnos sexualmente activos y con prácticas sexuales adecuadas 47 estudiantes $(61,0 \%)$ se caracterizan principalmente por tener más de 20 años de edad, 37 estudiantes $(78,7 \%)$, ser de sexo femenino, 32 estudiantes $(68,1 \%)$, de estado civil soltero 42 estudiantes $(89,4 \%)$, no haber tenido hijos 34 estudiantes (72,3\%), estudiar exclusivamente 34 estudiantes (72,3\%), tener un ingreso familiar mensual mayor a $\mathrm{S} /$. 1000,00 nuevos soles 32 estudiantes $(68,1 \%)$, y 25 estudiantes $(53,2 \%)$ estar cursando los dos últimos años de estudios.
Tabla $N^{\circ}$ 6: Distribución porcentual de los encuestados sexualmente activos según clasificación de relaciones sexuales y variables de estudio.

\begin{tabular}{|c|c|c|c|c|c|}
\hline \multirow{4}{*}{ Variables } & \multicolumn{4}{|c|}{$\mathrm{N}=77$} & \multirow{4}{*}{$\begin{array}{l}\text { Prueba de } \\
\text { hipótesis }\end{array}$} \\
\hline & \multicolumn{4}{|c|}{ Relaciones sexuales } & \\
\hline & \multicolumn{2}{|c|}{ Inadecuadas } & \multicolumn{2}{|c|}{ Adecuadas } & \\
\hline & \multicolumn{2}{|c|}{$\mathrm{n}=30$} & \multicolumn{2}{|c|}{$\mathrm{n}=47$} & \\
\hline Grupo etáreo (años). & $\mathrm{n}$ & $\%$ & $\mathrm{n}$ & $\%$ & $\mathrm{X}^{2}$ \\
\hline$<20$ & 10 & 33,3 & 10 & 21,3 & 1,38 \\
\hline$>20$ & 20 & 66,7 & 37 & 78,7 & $\mathrm{gl}: 1 ; p>0,05$ \\
\hline Sexo. & $\mathrm{n}$ & $\%$ & $\mathrm{n}$ & $\%$ & $\mathrm{X}^{2}$ \\
\hline Masculino & 8 & 26,7 & 15 & 31,9 & 0,24 \\
\hline Femenino & 22 & 73,3 & 32 & 68,1 & $\mathrm{gl}: 1 ; p>0,05$ \\
\hline Estado civil. & $\mathrm{n}$ & $\%$ & $\mathrm{n}$ & $\%$ & $\mathrm{X}^{2}$ \\
\hline Soltero & 28 & 93,3 & 42 & 89,4 & 0,35 \\
\hline Casado/unión libre & 2 & 6,7 & 5 & 10,6 & $\mathrm{gl}: 1 ; p>0,05$ \\
\hline Tiene hijo(s). & $\mathrm{n}$ & $\%$ & $\mathrm{n}$ & $\%$ & $\mathrm{X}^{2}$ \\
\hline Sí & 2 & 6,7 & 13 & 27,7 & 5,14 \\
\hline No & 28 & 93,3 & 34 & 72,3 & $\mathrm{gl}: 1 ; \mathrm{p}<0,05$ \\
\hline $\begin{array}{l}\text { Trabaja además de } \\
\text { estudiar. }\end{array}$ & $\mathrm{n}$ & $\%$ & $\mathrm{n}$ & $\%$ & $x^{2}$ \\
\hline Sí & 14 & 46,7 & 13 & 27,7 & 2,19 \\
\hline No & 16 & 53,3 & 34 & 72,3 & $\mathrm{gl}: 1 ; p>0,05$ \\
\hline $\begin{array}{ll}\text { Ingreso familiar } \\
\text { mensual } \\
\text { (S/.). }\end{array}$ & $\mathrm{n}$ & $\%$ & $\mathrm{n}$ & $\%$ & $x^{2}$ \\
\hline$<S / .1000 .00$ & 10 & 33,3 & 15 & 31,9 & 0,02 \\
\hline$>S / .1000 .00$ & 20 & 66,7 & 32 & 68,1 & $\mathrm{gl}: 1 ; p>0,05$ \\
\hline Año de estudios & $\mathrm{n}$ & $\%$ & $\mathrm{n}$ & $\%$ & $\mathrm{X}^{2}$ \\
\hline Primero & 0 & 0,0 & 6 & 12,8 & 47,03 \\
\hline Segundo & 4 & 13,3 & 11 & 23,4 & $g l: 5 ; p<0,05$ \\
\hline Tercero & 0 & 0,0 & 3 & 6,4 & \\
\hline Cuarto & 0 & 0,0 & 22 & 46,8 & \\
\hline Quinto & 26 & 86,7 & 5 & 10,6 & \\
\hline
\end{tabular}

\section{DISCUSION.}

La investigación incluyo 200 estudiantes de la Facultad de Enfermería de la Universidad Nacional "San Luis Gonzaga" de Ica, por lo tanto, se trabajó con adolescentes y jóvenes, en los cuales los procesos de maduración personal y social aún están por completarse; como se ha señalado este tipo de colectivos son ideales para identificar una amplia gama de estilos de vida, desde los adecuados hasta los inadecuados en un espectro grande de variabilidad en ambos sentidos.

Una alta proporción de los estudiantes pesquisados presentaron estilos de vida adecuados de acuerdo al cuestionario aplicado; estos hallazgos difieren significativamente con estudios similares realizados en estudiantes de enfermería por Rizo Z. en Alicante España (2). Triviño Z. en 
Colombia (6). Irazusta A. en el País Vasco España (3). Laguado J. en Colombia (4). Montero BA. en España (7). López J. en México (8) y Ponte A. en Lima Perú (5). Ya que hallaron estilos de vida poco adecuados.

En el estudio las conductas adecuadas se observaron en alumnos mayores de 20 años, mujeres, solteros, sin hijos, quienes estudiaban exclusivamente, con un ingreso familiar mensual por encima de la línea de pobreza (S/. 1000,00 nuevos soles) y quienes se hallaban cursando el último año de estudios. La prevalencia de relaciones sexuales fue de $38,5 \%$, con una tasa de $61,0 \%$ de conductas sexuales adecuadas; los alumnos sexualmente activos con conductas sexuales adecuadas presentaron las mismas características que las descritas en los estilos de vida globales. Según Marchan N, los comportamientos saludables varían de acuerdo al sexo de las personas, las mujeres tienen una incidencia de 2 a 3 veces mayor de sufrir el síndrome del intestino irritable y tiene unas 9 veces más de contraer enfermedades hepáticas (9).

Pero no sólo el género es un factor determinante y distinto en las actitudes $y$ comportamientos de la salud, también cobran importancia aspectos como: edad, nivel socioeconómico, experiencias pasadas, susceptibilidad y beneficios percibidos por cada persona. Considerando que la adolescencia y juventud son etapas decisivas en la adquisición y consolidación del estilo de vida, es prioritario estimular patrones maduros de comportamiento y el desarrollo de una identidad personal y social claramente definida. Pudiéndose lograr a través de las redes de apoyo familiar de las instituciones educativas y universidades (10).

Los estilos de vida individuales, caracterizados por patrones de comportamiento identificables, pueden ejercer un efecto profundo en la salud de un individuo y en la de otros. Si la salud ha de mejorarse permitiendo a los individuos cambiar sus estilos de vida, la acción debe ir dirigida no solamente al individuo, sino también a las condiciones sociales de vida que interactúan para producir y mantener esos patrones de comportamiento. Sin embargo, es importante reconocer que no existe un estilo de vida "óptimo" al que puedan adscribirse todas las personas. La cultura, los ingresos, la estructura familiar, la edad, la capacidad física, el entorno doméstico y laboral, harán más atractivas, factibles y adecuadas determinadas formas y condiciones de vida. Por ello, la estrategia de crear ambientes favorables para la salud se centra en gran medida en la necesidad de mejorar y cambiar las condiciones de vida para apoyar la salud (11).

El estilo de vida es uno de los mayores condicionantes sobre el estado de salud de la población y podría reducir la mortalidad global hasta en un $43 \%$. Una de las dimensiones más estudiadas de los estilos de vida es la actividad física, la cual se define como el conjunto de tareas motoras propias de una persona, que se realizan como parte de su actividad doméstica, laboral, escolar, recreativa y profesional; esta es una de las formas más sencillas de mejorar la salud y mantenerse sano. Ya que el ejercicio regular contribuye a controlar la presión arterial, fortalece los músculos y los huesos y mejora la función respiratoria y la actividad intestinal, además previene y controla ciertas enfermedades, como: padecimientos cardiovasculares, diabetes, obesidad y osteoporosis (8).

La actividad física, también favorece la auto estima, la relajación, las relaciones personales y la integración social, por medio del deporte en equipo que, si se orienta adecuadamente a través de la competencia, genera vínculos sociales, además de prevenir el consumo de tabaco, alcohol, drogas y la solución de conflictos por medios violentos (12).

Otra dimensión de los estilos de vida es la nutrición saludable; se considera que una alimentación es saludable cuando proporcionan los nutrientes necesarios para mantener la salud del organismo, la dieta saludable se caracteriza por el consumo de 
frutas (2-3 porciones), verduras (2-3 porciones), lácteos (2-3 porciones), proteínas (2-3 porciones) y carbohidratos (3-5 porciones), bajos en grasas y colesterol, así como limitar el consumo de alcohol, sal, café y azúcar, manteniendo un peso adecuado a través de la actividad física y la práctica de hábitos higiénicos al manipular los alimentos (7).

Es importante que el peso corporal se adecue en relación a la altura. Tener exceso de peso o ser obesa/o aumenta su riesgo de padecer enfermedades, en el sentido contrario, el estilo de vida de los jóvenes, puede conducir a comportamientos de riesgo y enfermedades crónicas. La publicidad engañosa, los regímenes de adelgazamiento mal programados para conseguir un canon de belleza, los horarios irregulares de las comidas, la compra y preparación de los alimentos, que en muchos casos pasan a ser ellos los encargados, etc., pueden convertirse en factores de riesgo nutricional. Todo ello, convierte a este grupo de población en un grupo especialmente vulnerable desde el punto de vista nutricional (13).

El consumo de tabaco, alcohol y otras drogas constituyen un problema prioritario de salud pública en los países desarrollados, ya que comporta niveles altos de morbi-mortalidad prevenibles (14).

La naturaleza multi causal del uso y abuso de drogas en los jóvenes justifica que las intervenciones preventivas se dirijan principalmente a la modificación de los factores de riesgo y protección. Los modos y actitudes seguidas en el consumo están relacionadas fuertemente con su personalidad, pero podrían generalizarse algunas, tales como: la grupalidad (el entorno de amigos influye positiva o negativamente, las pandillas actuales son más abiertas, flexibles e informales, esto permite a los jóvenes aguantar más tiempo en el grupo, el marco en el que se produce el mayor consumo). En un estudio sobre las consecuencias personales y sociales del consumo de drogas en jóvenes y adolescentes concluyeron que el consumo de estas sustancias disminuye la motivación, interfiere en los procesos cognitivos, contribuye a la aparición de desórdenes mentales e incrementa el riesgo de daño o muerte accidental. Igualmente, desde el punto de vista económico, el consumo de drogas puede conllevar una pérdida significativa de capital humano en la edad adulta $y$, en consecuencia, reduce la posibilidad de mayores salarios y rentas (15).

Los universitarios consideran tener conocimientos suficientes sobre sexualidad y anticoncepción, estando sus fuentes de información relacionadas con su entorno social y educativo y con menor importancia el personal sanitario y los padres. La edad de inicio de las relaciones sexuales coitales oscila entre $18 \pm 20$ años (17,8 años). Las prácticas sexuales más frecuentes son el coito vaginal y el sexo oral. Mayoritariamente utilizan método anticonceptivo, siendo el más conocido y usado el preservativo, aunque cerca del 30\% utiliza la píldora poscoital. Los varones inician antes las relaciones sexuales y tienen mayor número de parejas que las mujeres. El porcentaje de mujeres que necesitan estar enamoradas para mantener relaciones sexuales fue mayor que en los varones. Los varones mantienen relaciones sexuales bajo el efecto de drogas en mayor porcentaje que las mujeres.

Diferentes investigaciones han puesto de manifiesto la necesidad de realizar programas formativos que aborden no sólo la problemática del SIDA entre los jóvenes sino las relaciones interpersonales y sexuales en toda su amplitud, incluyendo el contagio de $\mathrm{VIH}$ como una de las consecuencias negativas del desconocimiento o las actitudes negativas hacia la sexualidad (16).

Algunos de estos estudios demuestran que la mitad de las conductas heterosexuales de los jóvenes sexualmente activos fueron adecuadas para protegerse frente al SIDA, 
otras enfermedades de transmisión sexual (ITS) y embarazos no deseados (usar siempre preservativo); un tercio de las conductas únicamente eran útiles para evitar embarazos (píldora anticonceptiva) y las restantes (usar ocasionalmente el preservativo o el coitus interruptus) les pusieron en riesgo frente a los tres problemas. Además, conforme se eleva la edad 0 el número de parejas de los estudiantes, es más frecuente el uso de píldoras anticonceptivas y menos probable el empleo de preservativo. Estos resultados sugieren que los estudiantes sexualmente activos están más interesados en la prevención de los embarazos no deseados que en evitar infectarse con el SIDA u otras ITS (17).

El ocio es, sin duda, uno de los fenómenos psicosociológicos más importantes y característicos del mundo occidental. Por su trascendencia creemos que se debe analizar desde una perspectiva multidimensional, teniendo en cuenta estereotipos, actitudes, prejuicios, relaciones intergrupales e interpersonales, calidad de vida, etc. un tiempo caracterizado por la libre elección y realización de actividades que satisfagan necesidades personales; y un tiempo cuya finalidad sea el descanso, la diversión, la creación o el desarrollo del sujeto (18). Si el trabajo está ligado a la civilización, el ocio se halla unido a la cultura, esto significa que genéricamente no se puede entender el ocio sin remitirse a lo cultural. Por último, respecto a la clasificación de prácticas culturales y de ocio, se ha desarrollado una tipología propia a partir de las realizadas por los diferentes enfoques teóricos. Dentro de las actividades de ocio se incluyen también el deporte, el turismo, el asociacionismo y el esparcimiento nocturno, tareas que aunque pueden comprender elementos culturales, éstos no son la base en sí misma de la propia actividad, también una serie de actividades generales que pueden desarrollarse al mismo tiempo que otras como charlar con los amigos, pasear y las que incluyen hobbies, juegos de mesa, de salón y juegos de azar.

Una dimensión importante de los estilos de vida son las relaciones interpersonales: el individuo está inmerso en la esfera social y, por lo tanto, busca relacionarse con sus pares. Las relaciones interpersonales constituyen un aspecto básico en la vida de un ser humano, funcionando no solo como un medio para alcanzar determinados objetivos; sino con un fin mismo, por lo que no son una tarea optativa o que pueda desarrollarse al azar. (19)

Las relaciones interpersonales en los profesionales de la salud constituyen un aspecto importante en su desempeño laboral, conductas que deben ser inducidas desde la etapa de formación profesional. Las relaciones interpersonales adecuadas facilitan actitudes positivas ante la vida, y en el trabajo permite el desarrollo de habilidades sociales, estimula la empatía con los usuarios, favorece actitudes y valores para afrontar conflictos, fracasos y frustraciones $y$, en definitiva, ayuda a generar climas de bienestar social y mantener estilo de vida saludable, traduciéndose en calidad y calidez humana en la atención y el servicio que se presta.

El manejo y reducción de las tensiones, es una pieza clave para mantener un óptimo estado de salud. El proceso o mecanismo general o sistema psico-neuro-endocrinológico, es el que se denomina con el término inglés de estrés.

Hans Selye, definió el estrés ante la Organización Mundial de la Salud como la respuesta no específica del organismo a toda demanda del exterior. La idea, nombre y concepto se han alojado fácilmente en la conciencia popular.

Estrés es la fuerza o el estímulo que actúa sobre el individuo y que da lugar a una respuesta, es la respuesta fisiológica o psicológica del individuo ante un estímulo ambiental, es una consecuencia de la 
interacción de los estímulos ambientales y la respuesta a la idiosincrasia del individuo. Técnicas como relajación, ejercicio físico y modificación de las situaciones que causan estrés son importantes para que los individuos permanezcan sanos.

\section{Correspondencia:}

Mg. Angélica Villarroel Huamaní

Correo electrónico: angelicavi@gmail.com

\section{REFERENCIAS BIBLIOGRAFICAS}

1. Vásquez E, Oblitas L. Promoción de los estilos de vida saludables. (Internet) Begoña Bogotá Colombia. Asociación Latinoamericana de Psicología de la Salud. Colombia. 2004. Citado 19 de Enero 2015. Disponible en: http://www.alapsa.org/detalle/0 5/index.htm

2. Rizo M, Baeza M, González N, Brauer G, Cortés E. Calidad de la dieta y estilos de vida en estudiantes de Ciencias de la Salud. Sociedad Española de Nutrición Hospitalaria. España. 2014. 29(1):153-157.

3. Irazusta AA, Ruiz LF, Gil OS, Gil GF, Irazusta AJ. Hábitos de vida de los estudiantes de enfermería. Universidad del País Vasco (CPV/EHU.EU de Enfermería). País Vasco. 2005. BIBLID.1137-439X. 27; 99-107.

4. Laguado JE, Gómez DMP. Estilos de vida saludable en estudiantes de Enfermería en la Universidad Cooperativa de Colombia. Rev Colombia 2014. 19(1):68-83.

5. Ponte A. Estilo de vida de los estudiantes de enfermería, según año de estudio de la Universidad Nacional Mayor de San Marcos. Tesis Pre grado. Lima Perú. Facultad de Medicina, EAP. Enfermería. Universidad Nacional Mayor de San Marcos. 2010.

6. Triviño Z, Vargas G. Conductas promotoras de salud en estudiantes de una institución de educación superior. Bogotá Colombia. Rev Aquichan. 2012; 12 (3): 275-285.
7. Montero BA, Úbeda MA, García GA. Evaluación de los hábitos alimentarios de una población de estudiantes universitarios en relación con sus conocimientos nutricionales. España. Rev. Sociedad Española de Nutrición Hospitalaria. 2006; 21(4): 466-73

8. López J, Gonzales $\mathbf{M}$, Rodríguez $\mathbf{M}$. Actividad física en estudiantes universitarios: prevalencias, características y tendencia. (Internet). En Medigrafic Artemisa. Rev. México 2006. 22(3) (citado 19 de Julio 2014). Disponible en: http://new.medigraphic.com/cgibin/resumen.cgi?IDREVISTA=83\&IDARTI CULO $=14423 \&$ IDPUBLICACION $=1480 \& N$ OMBRE=Medicina\%20Interna\%20de\%20 $M C \% 20 B$

9. Marchan N, Ramírez H, López J. Estilos de vida de los estudiantes del Programa de Enfermería de la Universidad Centro occidental "Lisandro Alvarado" periodo junio - noviembre. Tesis Pre grado. Barquisimeto Venezuela. Programa de Enfermería, Universidad Centro Occidental Lisandro Alvarado; 2005.

10. O’Connel S, Bare B. Enfermería Médico Quirúrgica. 10ª ed. México. Ed. Mc Graw Hill Interamericana, 2005. p. $60-61$.

11. Marqués $M$, Cabezas $P$, Camarelles $G$, Córdoba G, Gómez P, Muñoz S, et al. Recomendaciones sobre el estilo de vida. (Internet) Barcelona: Sociedad Española de Medicina de Familia y Comunitaria. España. 2009. (Citado 12 de Diciembre 2014) Disponible en: http://www.papps.org/upload /file/08PAPPSACTUALIZACION2009.p

12. Lumbreras $D$, Moctezuma $A$, Dosamantes C, Medina H, Cervantes R, López $L$, et al. Estilo de vida y riesgos para la salud en estudiantes universitarios: hallazgos para la prevención. México. Rev. Digital Universitaria UNAM 2009. 10(2):1-XX-14XX.( citado 10 de Diciembre 2014) Disponible en: http://www.revista.unam. mx/vol.10/num2/art12/int12.htm 
13. Córdoba A, Carmona G, Terán V, Márquez M. Relación del estilo de vida y estado de nutrición en estudiantes universitarios. Rev. Medwave 2013; 13(11):5864 doi: 10.5867/medwave. 2013;11.5864. Disponible en: http://www.medwave.cl/link.cgi/Medwave/E studios/Investigacion/5864

14. Bogdanovica I, Godfrey F, Mc Neill A, Britton J. Smoking prevalence in the European Union: a comparison of national and transnational prevalence survey methods and results. Tob Control. European Union. 2011: 20(1):e4.

15. Organización Mundial de la Salud. Informe Mundial de Situación sobre Alcohol y Salud. Ginebra. Suiza. 2014.

16. Federación Española de Sociedades de Sexología. La conducta sexual. (Internet) España. (Citado el 10 de Enero 2015) Disponible en: http://www.fess.org.es/conductasexual.php.

17. Bandura A. Social cognitive theory and exercise of control over HIV infection. In R. Di Clemente \& R. J. Peterson (Eds.), Preventing AIDS: Theories and methods of behavioral interventions (pp. 25-60). New York: Plenum. 1994.

18. Instituto Nacional de la Juventud. Sexta Encuesta Nacional de la Juventud (Internet). Santiago Chile 2009. (Citado el 1 de Agosto 2014) Disponible en: www.injuv http://.gob.cl
19. Cabezas P, Camarelles G, Córdoba G, Gómez $P$, Muñoz $S$, et al. Recomendaciones sobre el estilo de vida. (Internet) Sociedad Española de Medicina de Familia y Comunitaria. Barcelona 2009. (Citado el 12 de Diciembre 2014) Disponible en: $\quad$ http://www.papps.org lupload/file/08PAPPSACTUALIZACION200 9.p

Recibido: 13/02/16 Aprobado para Publicación: 15/04/2017 